\title{
Image Retrieval Based on HSV Feature and Edge Direction Feature Yanxue DONG ${ }^{1, a}$ \\ ${ }^{1}$ School of Information, Linyi University, Linyi, 276000, China \\ aemail: dyxtmb@163.com
}

Keywords: CBIR; HSV; Edge Direction;

\begin{abstract}
With the development of multimedia and Internet technology, content-based image retrieval (CBIR) systems have been widely used. However, how to automatically detect and target images in an image database still remains challenging. Since the HSV color space is more benefiting with human visual perception mechanism, we convert the image from RGB color space to HSV color space firstly, obtain the color structure map and edge direction index map and then get the color feature and edge direction feature, finally accomplish the retrieval. Experimental results in Corel_10000 data set show that the proposed algorithm performs better both on precision rate and recall rate compared with two classic algorithms of image retrieval.
\end{abstract}

\section{Introduction}

With the development of digital image technology, image database is getting larger and larger, how to query and retrieve images we want from so large amount of images has become a research hotspot in the field of information processing.

The process of content-based image retrieval(CBIR) can generally be divided into the following steps:

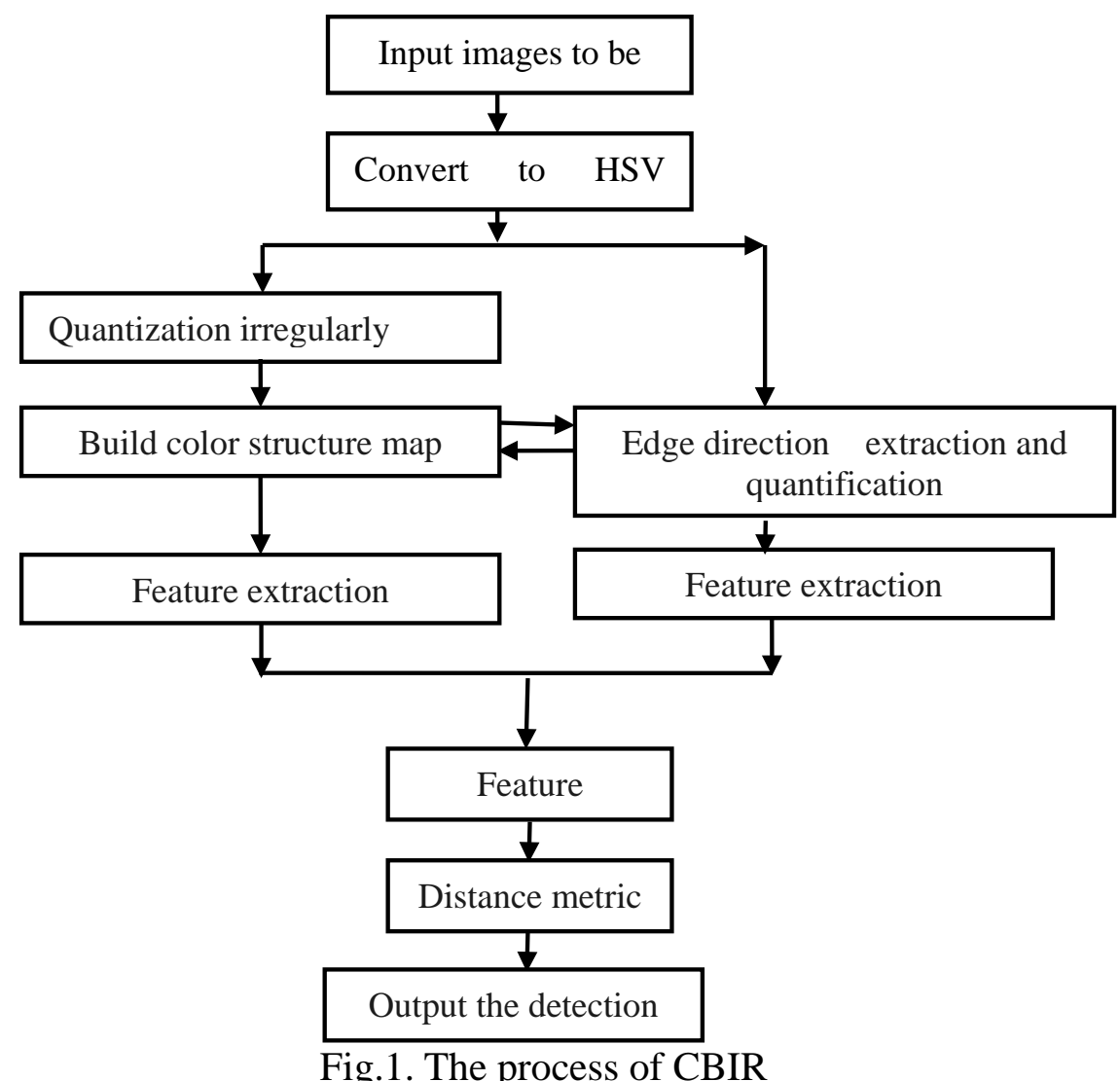

1. Pre-analysis, applying appropriate algorithms to extract and store low-level features of each image, the system should extract the feature of the image be submitted by the user to retrieve images applying the same algorithm;

2. Select suitable algorithm to measure similarity, make feature similarity measure on feature of 
image to be retrieved and feature of images in feature database.

3. Find images similar with image to be retrieved, and show them to the users in descending order of similarity.

The retrieval process mentioned in this paper is shown in Fig.1, first, input the image to be retrieved, transfer the RGB color space to HSV color space because the HSV color space is more in line with human visual perception mechanism, and then, non-equal interval quantization

and extraction and quantization of edge direction in HSV color space, and finally, extraction and fusion of features using suitable distance metric scheme, output the images similar to sample image the system find.

\section{RGB Color Space to HSV Color Space}

Color information is a kind of underlying and intuitive physical feature and it has strong robustness for image noise, size, and orientation.

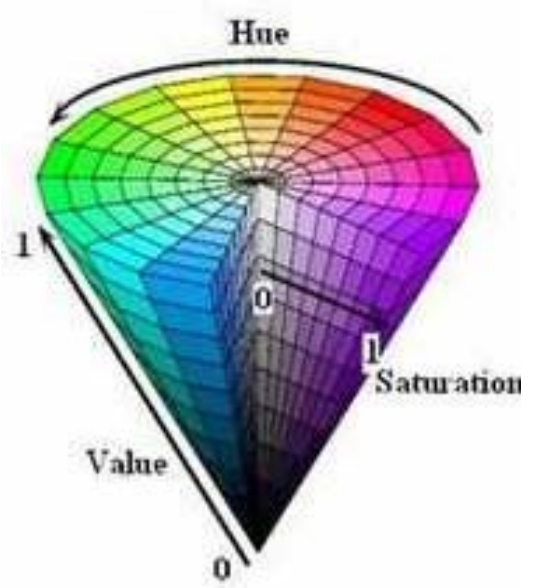

Fig.2. HSV color space

Compared to RGB color space, HSV color space is more consistent with human visual perception mechanism ${ }^{[1]}$, and its color space model is as shown in Fig.2, where Hue $(\mathrm{H})$ refers to the basic properties of color, especially affects human' $\mathrm{s}$ visual judgment, it ranges from $0^{0}$ to $360^{\circ}$; Saturation (S) refers to the color purity, i.e. having a degree of white color. The bigger the value of $\mathrm{S}$, the higher color purity, the range of $\mathrm{S}$ is $0-1$; Value $(\mathrm{V})$ refers to the brightness and it ranges from 0 to 1.

The advantages of HSV color space are shown as follows :

1. Because the three components of HSV color space are independent and so the human eye can perceive the three color components individually

2. The human eye is more sensitive to changes in brightness, than to changes in color depth , so the HSV color space is more consistent with human visual perception than RGB color space;

3. HSV space is a uniform color model, the distance between colors in human visual system is proportional to the Euclidean distance in HSV color space, that is, when the value of Euclidean distance is small, the color differences human eyes feel are small and when the value of Euclidean distance is large, the color differences the human eyes fell is great.

\section{Non-equal interval quantization to the color}

According to the perception characteristics of color of human visual, make non-equal interval 
quantization to the three components: $\mathrm{H}, \mathrm{S}$ and $\mathrm{V} . \mathrm{H}$ is quantized into eight levels, $\mathrm{S}$ and $\mathrm{V}$ are all quantized into 3 levels. Thus, we get the quantized color index map $\mathrm{C}(\mathrm{x}, \mathrm{y})$, the index value ranges 0-71. Detailed quantization scheme as follows:

$$
H=\left\{\begin{array}{l}
0, H \in[0,25] \cup(335,360] \\
1, H \in(25,37] \\
2, H \in(37,70] \\
3, H \in(70,160] \\
4, H \in(160,200] \\
5, H \in(200,275] \\
6, H \in(275,295] \\
7, H \in(295,335]
\end{array}\right.
$$

$$
\begin{gathered}
S=\left\{\begin{array}{l}
0, S \in[0,0.37] \\
1, S \in(0.37,0.76] \\
2, S \in(0.76,1]
\end{array}\right. \\
V=\left\{\begin{array}{l}
0, V \in[0,0.37] \\
1, V \in(0.37,0.76] \\
2, V \in(0.76,1]
\end{array}\right.
\end{gathered}
$$

Fig.3. Quantization to H, S, V

\section{Extraction and quantification of the edge direction}

Shape and contours information of images can be well described by edge direction. In this paper, a simple and effective method for detecting edge direction is applied. For an image, assume that a point's vector is $a=\left(H_{x}, S_{x}, V_{x}\right), b=\left(H_{y}, S_{y}, V_{y}\right)$, where $H_{x}, S_{x}$ and $V_{x}$ denote gradient of $H, S$ and $\mathrm{V}$ in the horizontal direction, $\mathrm{H}_{\mathrm{y}}, \mathrm{S}_{\mathrm{y}}$, and $\mathrm{V}_{\mathrm{y}}$ denote gradient of $\mathrm{H}, \mathrm{S}$ and $\mathrm{V}$ in the vertical direction. The Sobel operator are used to accomplish this process ${ }^{[2]}$. Calculate the dot product of a and $\mathrm{b}$ as follows:

$$
\begin{aligned}
& |a|=\sqrt{\left(H_{x}\right)^{2}+\left(S_{x}\right)^{2}+\left(V_{x}\right)^{2}} \\
& |b|=\sqrt{\left(H_{y}\right)^{2}+\left(S_{y}\right)^{2}+\left(V_{y}\right)^{2}} \\
& a \cdot b=H_{x} \cdot H_{y}+S_{x} \cdot S_{y}+V_{x} \cdot V_{y}
\end{aligned}
$$

The angel between a and $\mathrm{b}$ can be obtained:

$$
\begin{aligned}
& \cos (a, b)=\frac{a \cdot b}{|a| \cdot|b|} \\
& \theta(x, y)=\arccos \left[\frac{a \cdot b}{|a| \cdot|b|}\right]
\end{aligned}
$$

Calculate the angle value of every pixel in an image, quantize angle values in 18 levels, the interval is equal and its value is $10^{\circ}$, thus, the index map $\theta(x, y)$ of edge direction is obtained.

\section{Construction of the color chart}

Color chart can be constructed according to the color index map :

(1) Color index map C(x,y) is processed applying template shown in Fig.4 


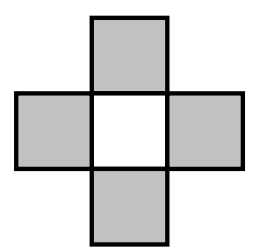

Fig.4. The center point and its neighbor field

(2) As shown in Fig.4, white dots in the middle represents the center point, the gray pixel represents the neighbors domain of center point. In the area the template correspond, find the neighbors domain of center point, if one or more values of the neighborhood domain are equal to the value of center point, the value of this template at all positions are reserved, if no value of neighbor domain is equal to center point, the value of the template at all locations are set 0 .

The specific process is shown in Fig.5, (a) is a part of a color index map, (b) and (c) show two cases when the $3 \times 3$ template travels on the color index map shown in (a). As shown in (a), in the area the $3 \times 3$ template corresponds, one point in the neighbor domain of the center point has the same value as center point, thus the value of the area the $3 \times 3$ template corresponds is reserved. As shown in (c), no point in the neighbor domain of the center point has the same value as center point, thus the value of the area is set 0 , and then we get the color chart $\mathrm{T}(\mathrm{x}, \mathrm{y})$, as shown in (d).

\begin{tabular}{|l|l|l|l|l|l|}
\hline 1 & 3 & 2 & 0 & 2 & 5 \\
\hline 3 & 5 & 4 & 3 & 4 & 0 \\
\hline 2 & 5 & 2 & 4 & 3 & 1 \\
\hline 0 & 2 & 5 & 0 & 3 & 1 \\
\hline 2 & 1 & 3 & 1 & 2 & 2 \\
\hline 5 & 0 & 2 & 3 & 2 & 0 \\
\hline
\end{tabular}

(a)

\begin{tabular}{|l|l|l|l|l|l|}
\hline 1 & 3 & 2 & 0 & 2 & 5 \\
\hline 3 & 5 & 4 & 3 & 4 & 0 \\
\hline 2 & 5 & 2 & 4 & 3 & 1 \\
\hline 0 & 2 & 5 & 0 & 3 & 1 \\
\hline 2 & 1 & 3 & 1 & 2 & 2 \\
\hline 5 & 0 & 2 & 3 & 2 & 0 \\
\hline
\end{tabular}

(b)

\begin{tabular}{|l|l|l|l|l|l|}
\hline 1 & 3 & 2 & 0 & 2 & 5 \\
\hline 3 & 5 & 4 & 3 & 4 & 0 \\
\hline 2 & 5 & 2 & 4 & 3 & 1 \\
\hline 0 & 2 & 5 & 0 & 3 & 1 \\
\hline 2 & 1 & 3 & 1 & 2 & 2 \\
\hline 5 & 0 & 2 & 3 & 2 & 0 \\
\hline
\end{tabular}

(c)

\begin{tabular}{|l|l|l|l|l|l|}
\hline 1 & 3 & 2 & 0 & 0 & 0 \\
\hline 3 & 5 & 4 & 0 & 0 & 0 \\
\hline 2 & 5 & 2 & 0 & 0 & 0 \\
\hline 0 & 0 & 0 & 0 & 3 & 1 \\
\hline 0 & 0 & 0 & 1 & 2 & 2 \\
\hline 0 & 0 & 0 & 3 & 2 & 0 \\
\hline
\end{tabular}

(d)

Fig.5 


\section{Feature representation}

After above operation, we get color chart $\mathrm{T}(\mathrm{x}, \mathrm{y})$ and its index value can be expressed as $p \in\{0,1, \ldots, P-1\}$. For every point $\left(x_{0}, y_{0}\right)$ in color chart $\mathrm{T}(\mathrm{x}, \mathrm{y})$, its index value is $T\left(x_{0}, y_{0}\right)=p_{0}$, index value of every point's neighbor domain is expressed $T\left(x_{i}, y_{i}\right)=p_{i},(i=1,2,3,4)$. For edge direction chart $\mathrm{O}(\mathrm{x}, \mathrm{y})$, index value of every point is $q \in\{0,1, \ldots, Q-1\}$, point $\left(x_{0}, y_{0}\right)$ in edge direction index map is described as $\mathrm{O}\left(x_{0}, y_{0}\right)=\boldsymbol{q}_{0}$, its neighbor domain is described as $O\left(x_{i}, y_{i}\right)=q_{i},(i=1,2,3,4)$. In this paper, the value of $\mathrm{P}$ is 71 and the value of $\mathrm{Q}$ is 17 . Then we describe the color feature and edge direction feature as follows:

$$
\begin{aligned}
& H_{\text {color }}(T(x, y))=\sum_{i=1}^{N(T(x, y))} N_{i}(T(x, y)) \\
& H_{\text {ori }}(O(x, y))=\sum_{i=1}^{N(O(x, y))} N_{i}(O(x, y))
\end{aligned}
$$

Where $\mathrm{N}($.) indicates the amount of units which have index value of $\mathrm{p}$ or $\mathrm{q}$ in color or edge direction chart. $\mathrm{Ni}($.$) indicates the amount of dots which has the same value as the value of its neighbor$ domain. $\mathrm{H}_{\text {color }}(\mathrm{T}(\mathrm{x}, \mathrm{y}))$ indicates color feature vector with 72 dimension we finally get, $\mathrm{H}_{\text {ori }}(\mathrm{O}(\mathrm{x}, \mathrm{y}))$ indicates edge direction feature vector with 18 dimension, thus, a feature vector with 90 dimension is used to retrieve images.

\section{Feature weighting}

$$
H=\left\lfloor\partial H_{\text {color }}(T(x, y)),(1-\partial) H_{\text {ori }}(O(x, y))\right\rfloor
$$

According to the attribute of the images, the two characteristics should have different weights. First, give the color feature weights $\alpha$, correspondingly, the weight of edge direction feature is $1-\alpha$. as shown in Equation (8). The weight $\alpha$ has step size of 0.01 and it ranges from 0 to 1, a new feature $\mathrm{H}$ would be used to retrieve images which is formed by two weighted feature, i.e. Structure Feature Histogram(SFH).

\section{Conclusion}

In this paper, similarity is measured applying Equation (9). Where $\mathrm{K}$ indicates the image to be retrieved and all images in data set have feature vector with $\mathrm{K}$ dimension, the feature vector of the image $\mathrm{Q}$ to be retrieved is described as $\mathrm{Q}=[\mathrm{Q} 1, \mathrm{Q} 2, \ldots, \mathrm{QK}]$, the feature vector of every image $\mathrm{T}$ in data set is described as $\mathrm{T}=[\mathrm{T} 1, \mathrm{~T} 2, \ldots \mathrm{TK}]$. The distance of $\mathrm{Q}$ and $\mathrm{T}$ is

$$
D(Q, T)=\sum_{i=1}^{k}\left|\frac{Q_{i}-T_{i}}{1+Q_{i}+T_{i}}\right|
$$

In this paper, the value of $\mathrm{K}$ is 90 , that is every image has feature vector with 90 dimension, the smaller the value of $\mathrm{D}(\mathrm{Q}, \mathrm{T})$, the more similar image $\mathrm{Q}$ and image in data set.

Two experiments are used to validate the algorithm proposed in this paper. Experiment 1, select 10 class of images randomly from corel_10000 data set, test the impact of retrieval performance on different color space, the results are shown in Tab.1 which indicate that in HSV color space, the precision rate and recall rates are all higher than those in RGB color space. Experiment 2, select 10 classes of images randomly from corel_10000 data set, test the impact of retrieval performance on 
different feature weights, the results are shown in Tab.2. The two experiments all show that the algorithm proposed in this paper has better performance.

Tab.1. Comparison of different color spaces.

\begin{tabular}{|c|c|c|}
\hline & RGB color space & HSV color space \\
\hline Precision Rate(\%) & 74.47 & 78.32 \\
\hline Recall Rate(\%) & 8.56 & 9. \\
\hline
\end{tabular}

Tab.2. Comparison of different weights.

\begin{tabular}{|c|c|c|}
\hline & $1: 1$ & $6: 94$ \\
\hline Precision Rate(\%) & 72.61 & $82 . .45$ \\
\hline Recall Rate(\%) & 8.35 & 9.83 \\
\hline
\end{tabular}

\section{Acknowledgments}

This work was supported by Shandong Provincial Natural Science Foundation (ZR2014FL022), China.

\section{References}

[1] Gonzalez R C, Woods R E. Digital image processing[J]. 2002, 9(7):824-849.

[2] Liu G H, Yang J Y. Image retrieval based on the texton co-occurrence matrix[J]. Pattern Recognition, 2008, 41(12): 3521-3527.

[3] Murala S, Maheshwari R P, Balasubramanian R. Local tetra patterns: a new feature descriptor for content-based image retrieval[J]. Image Processing, IEEE Transactions on, 2012, 21(5): 2874-2886.

[4] Liu G H, Li Z Y, Zhang L, et al. Image retrieval based on micro- structure descriptor[J]. Pattern Recognition, 2011, 44(9): 2123-2133.

[5] Chen Y K, Wong E K. 1999. Augmented image histogram for image and video similarity search. SPIE, 3656:523 532. 